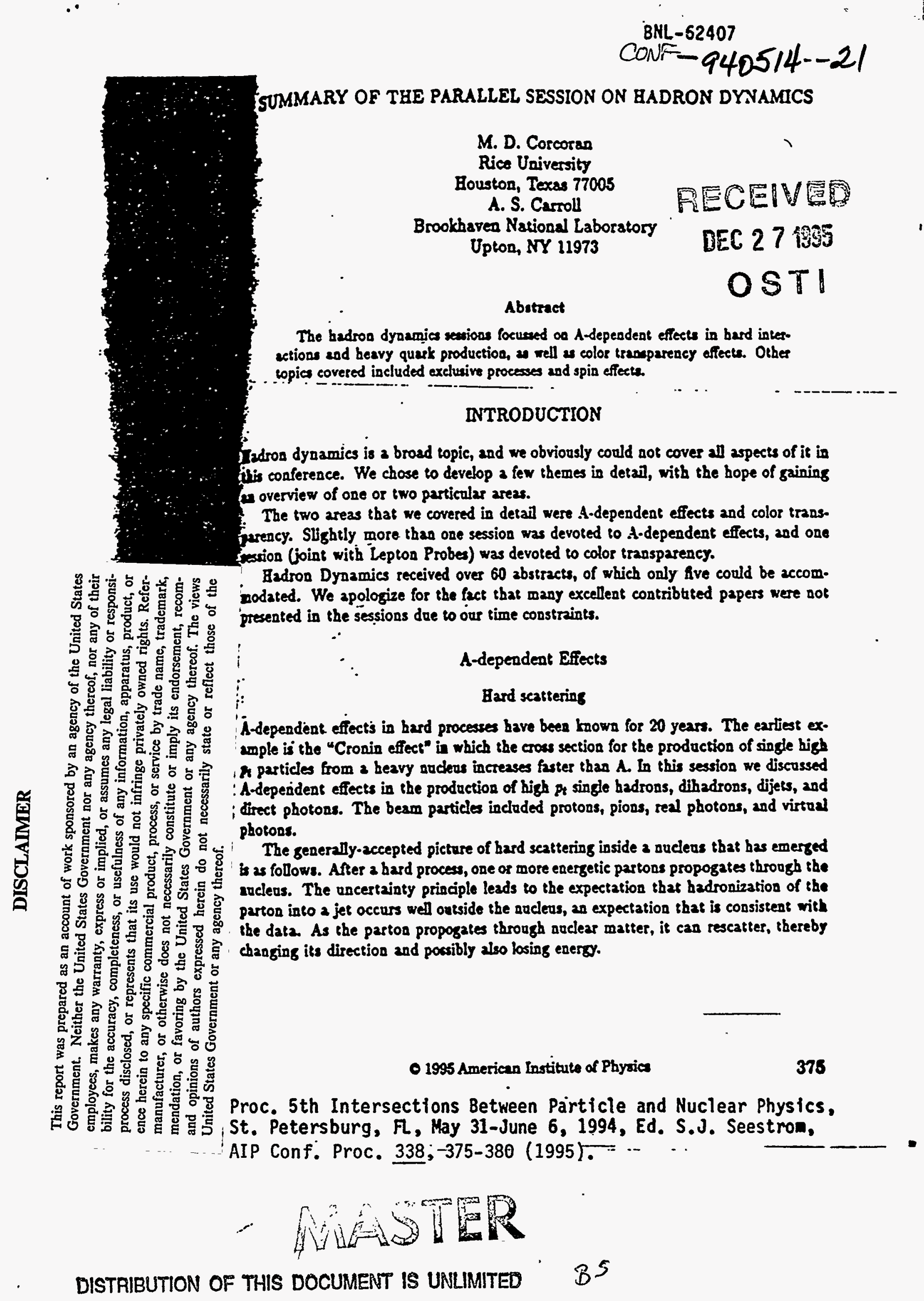


Traditionally A-dependent effects have been characterized by the parameter $\alpha$ in the expression $\sigma(p A)=\sigma(p p) A^{\alpha}$. If all of the nucleons act independently $\alpha$ would be 1 . If the cross section falls steeply with respect to the variable being considered (such as $p_{t}$ ), smearing of the angle of the outgoing parton will lead to $\alpha>1$. A value of $\alpha$ less than one indicates shadowing of some sort.

Another way to study nuclear rescattering is through its contribution to the unbal. ance in $p_{t}$ of the outgoing particles in dihadron or dijet final states. Define $\overrightarrow{k_{t}}$ as the vector unbalance in $p_{t}$ of the dijet or dihadron system. $\vec{k}_{t}$ has contributions from several sources, such as primordial Fermi motion, gluon radiation, and hadronization, as well as experimental effects. When the target is a heavy nucleus, nuclear rescattering can make a contribution to $\vec{k}_{t}$. A variable in recent use to quantify nuclear rescattering effects is $k_{t \phi}$, the $\phi$ component of $\vec{k}_{t}$ in the transverse plane (see figure 1 ).

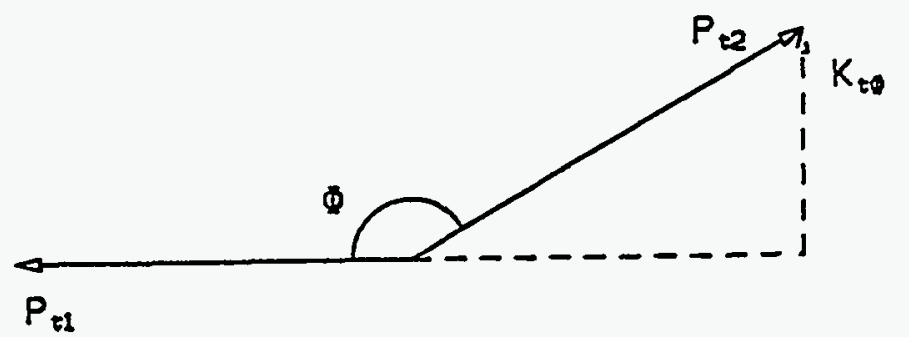

Figure 1. Definition of $k_{t \phi}$.

Tom Fields of Argonne National Laboratory began the session on A-dependent effects with a review of hard-scattering data. The original Cronin data has been confirmed and extended to higher $p_{t}$ and CM energies by several Fermilab experiments. Even though many experiments have been done, in general these effects are not well mappedout as functions of $p_{t}$ and especially energy. In addition to single high $p_{t}$ hadrons, several experiments have looked at $\mathrm{A}$-dependent effects in the production of high $p_{t}$ dihadron pairs. However, most of the dihadron experiments suffered from limited acceptance in azimuth angle $\phi$, which as described by Fields, affects the measurement of $\alpha$. [1]

Two experiments looked at A-dependent effects in dijet production in pA interactions, Fermilab E557 and E609. Both experiments saw substantial effects. E609 has published results on $k_{t \delta}$ for dijets, indicating a large smearing of the opening angle between the jets $(\Delta \phi)$ due to the presence of the nucleus (Figure 2 [2]). From this plot it is clear why an experiment with limited acceptance in $\phi$ would measure a different value of $\alpha$ than one with full acceptance.

C. C. Chang from Maryland presented results for Fermilab E683. This experiment used a real photon beam with mean energy $250 \mathrm{GeV}$ to study jet production. $k_{t 0}$ shows a definite nuclear contribution, see figure 3 [3]. A similar nuclear contribution is seen for dijets produced in a pion beam. An energy dependence of the nuclear contribution to $k_{t \phi}$ was also shown. 
:terized by the parameter ct independently $\alpha$ would bo iable being considered (such ad to $\alpha>1$. A value of $a$ 1 its contribution to the win i final states. Define $\overrightarrow{k_{t}}$ as th has contributions from severe 1 , and hadronization, as well nuclear rescattering can mabs - nuclear rescattering effects gure 1).

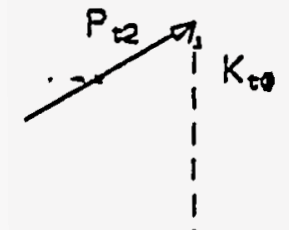

a session on A-deyendent efJnin data has been confirmed ermilab experiments. Even effects are not well mappedIgle high $p_{t}$ hadrons, several duction of high $p_{t}$ dihadron . from limited acceptance in measurement of $\alpha$. [1] $t$ production in $\mathrm{pA}$ interac:pstantial effects. $\mathrm{E609}$ has ng of the opening angle beigure 2 [2]). From this plot , would measure a different

lab E683. This experiment $y$ jet production. $k_{t \phi}$ shows luclear contribution is seen of the nuclear contribution

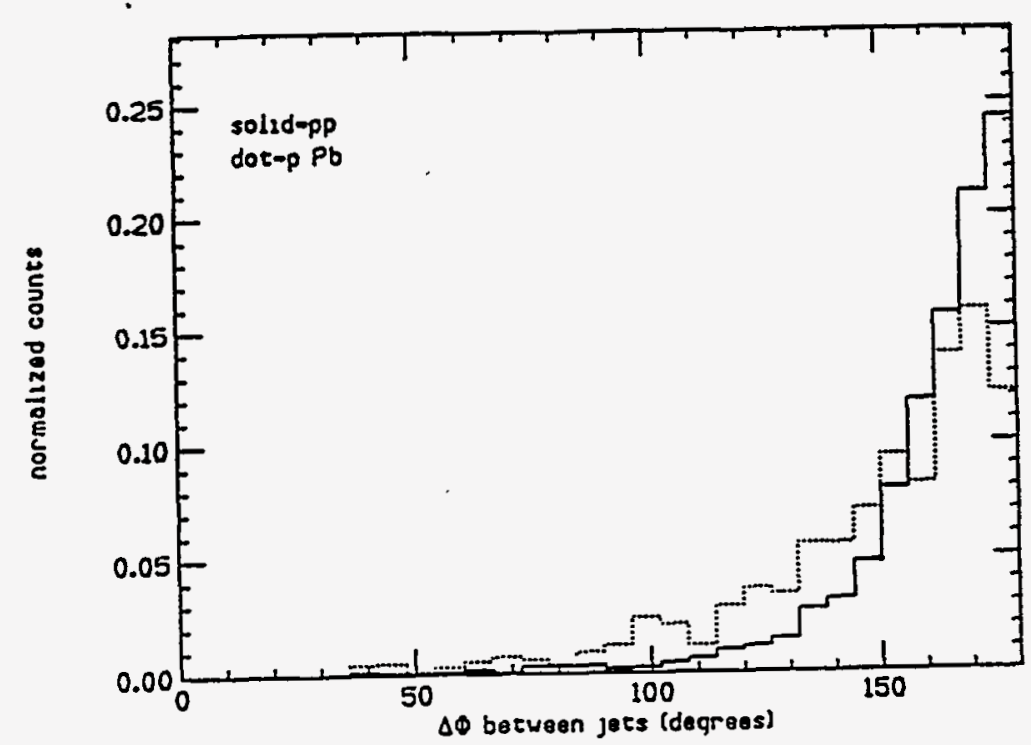

Figure 2. E609 data showing the broadening of the opening angle between the jets $\Delta \phi$ due to the presence of a heavy nucleus.

M. Zielinski from Rochester presented results from Fermilab E706 on the Adependence of high $p_{t}$ hadron and direct photon production in $\pi A$ interactions. E706 results on pion production agree with the earlier data at lower energy of Frisch et al., $\alpha_{\pi}=1.08$. E706 sees a similar value of $\alpha$ for single high $p_{t} \eta$ production. The interesting new result is that direct photon production is consistent with $\alpha=1$. It would be expected that the outgoing photon would not undergo any multiple scattering in the nuclear medium, so this result is consistent with that expectation. ET06 compared their dijet results on $k_{t d}$ with those of $\mathrm{E} 683$. They are quite similar, with E706 perhaps seeing a somewhat stronger A-dependence.

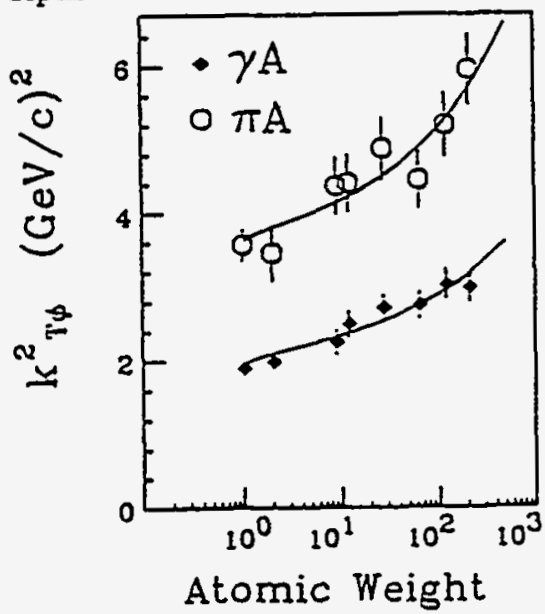

Figure 3. E683 data showing the increase of $k_{l 0}$ with increasing A. 
The $\mathrm{A}$-dependent effects seen in $\mathrm{pA}, \pi \mathrm{A}$ and $\gamma \mathrm{A}$ interactions are in contrast to the well-known lack of A-dependent effects in Drell-Yan production, where it has bee known for several years that $\alpha=1$. One could interpret the data to indicate that there is substantial multiple scattering in the nucleus in the final state but not the initial state. It is a long-standing puzzle in this field as to why that would be the case.

Bruce Baller of Fermilab presented results for deep inelastic scattering from a 500 $\mathrm{GeV}$ muon beam, Fermilab E665. In the region $x_{b j}<.01$, the so-called shadowing region, the cross section ratios of heavy nuclei to deuterium are less than one. E665 sees a dependence of this ratio on the fraction of the available energy observed in the detector, the first time shadowing has been seen to depend on details of the hadronic final state. E665 has studied both $1+1$ jet events (a single hard-scattered parton plus a target jet) and $2+1$ jet events (two hard partons plus a target jet). For the $2+1$ jet sample, E665 studies $k_{t \phi}$, and they do not see a nuclear enhancement. This result is in contrast to E683 results discussed above from a real photon beam and is another (new) puzzle.

\section{Heavy Quark Production}

Results were presented from Fermilab Ei72 and E789 on the A-dependence of heavy quark production. The $\mathrm{J} / \psi$ suppression is by now well-known. In $\pi A \rightarrow \mathrm{J} / \psi \rightarrow \mu \mu, \alpha$ is less than one and falls with increasing Feynman $x$. The same effect has now been seen for $\psi^{\prime}$ and $\Upsilon$ (1s) production. However, both E789 and another Fermilab experiment, E769, agree that $\alpha$ is equal to one within errors for open charm (D meson) production.

The difference in the A-dependence of $J / \psi$ production and Drell-Yan production has generated a great deal of theoretical work. Ideas discussed included parton energy loss coupled with a steeply falling Feynman $x$ distribution. But it is safe to say that, on the theoretical side, there was no consensus.

\section{Theoretical Contributions}

Boris Kopeliovich from JINR, Dubna presented a calculation which linked $k_{t \phi}$ and $\alpha$ in pA interactions. The model is in qualitative but not quantitative agreement with E609 data.

John Ralston of Kansas gave the theoretical review A-dependence effects. At this time no theoretical model can successfully describe all of the existing experimental results, or even a substantial fraction of them. However, recent theoretical interest in this area gives one reason to believe progress will be made in the near future.

\section{Color Transparency}

If a hadron is to pass through a nucleus and stay intact, it must be a "small" hadron. A small color dipole should have small interactions and therefore a good chance of escaping a nucleus intact. The probability that a hadron passes through a nucleus intact is the transparency. A small size implies a large momentum transfer, so one expects the transparency to increases as $Q^{2}$ increases. Color transparency is closely related to the QED effect called the Landau-Pomeranchuk-Migdal effect, in which the 
A interactions are in contrats. an production, where it has et the data to indicate that he final state but not the inf hy that would be the case. :p inelastic scattering from 2 $:<.01$, the so-called shadow aterium are less than one $E$ available energy observed in epend on details of the hadro ingle hard-scattered parton of us a target jet). For the $2+1$ .r enhancement. This result in totọn beam and is another (new

$$
\text { - = }
$$

on the A-dependence of hear? -known. In $\pi A \rightarrow \mathrm{J} / \psi \rightarrow \mu \mu$, te same effect has now been seen 1 another Fermilab experiment, n charm ( $D$ meson) production. in and Drell-Yan production has sed included parton energy loss Hut it is safe to say that, on the

;

Ition which linked $k_{t \phi}$ and $\alpha$ in tntitative agreement with E609

A-dependence effects. At this $I$ of the existing experimental $\because$, recent theoretical interest in de in the aear future.

it must be a "small" hadron. $d$ therefore a good chance of con passes through a nucleus : momentum transfer, so one Color transparency is closely ik-Migdal effect, in which the ination from an energetic electron is suppressed if the formation time for the radiated Woton is longer than the mean free path for another scatter.

Several experinents showed results from searches for color transparency. John Zumfrom Los Alamos showed data from LAMPF experiments E1136 and E1174, with f.to $\mathrm{MeV}$ pions in the reaction $\mathrm{C}\left(\pi, \pi^{\prime}\right)$. Internuclear Cascade Models (INC) greatly unHrestimate the number of pions in certain regions of phase space, suggesting the idea Fat color transparency might be a possible mechanism to enhance pion production.

iy. B. Filippone from California Institute of Technology presented results from SLAC IE18, $\mathrm{A}\left(e, e^{\prime} p\right)$, with the proton being knocked out of the nucleus and escaping intact. The expectation from color transparency is that the probability for such an interaction would increase as $Q^{2}$ increases, but no significant effect was observed.

3-Y Wu from Penn State presented results from experiments at the AGS which are looking for color transparency effects in $p N \rightarrow p p(s N-1)$, with no other particles produced in the event. The color transparency signal would be a single proton knocked ont of the nucleus and escaping intact without interacting. In older E834 data, this group saw an interesting rise in transparency with incident momentum, followed by a drop as the momentum continued to increase. The rise is consistent with a a simple model of color transparency in this system, but the drop is not. Presumably some amplitudes other than the perturbative amplitudes are involved. This group is taking data with a new detector, EVA, during the current run at BNL, so new results are expected shortly.

G. Fang from Harvard presented results for exclusive $\rho$ production from Fermilab 2665. If the $\rho$ is produced at large $Q^{2}$, it should be small and therefore should have a good chance to escape the nucleus. Statistics are limited, but a rise in transparency with $Q^{2}$ is seen, consistent with theoretical expectations, see figure 4 [4].

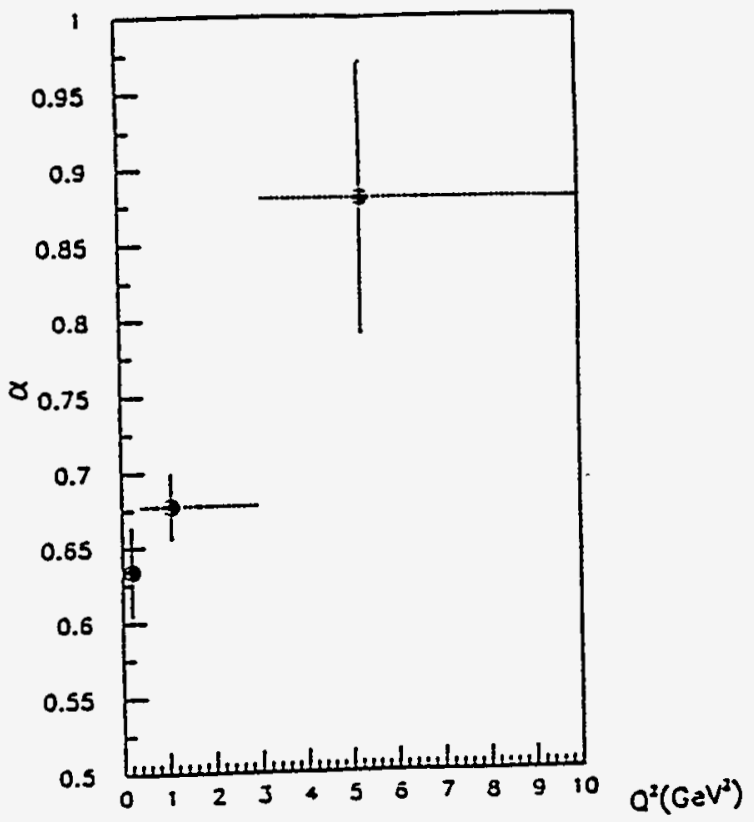

Figure 4. $\alpha$ vs $Q^{2}$ for exclusive $\rho$ production in E665. The prediction of color transparency is that $\alpha$ should approach 1 as $Q^{2}$ increases. 


\section{Parallel Session on Hadron Dynamics}

Mark Strikman from Penn State gave a theoretical summary, insisting that the theoretical underpinnings of the color transparency idea are well established, even if the experimental situation is not so clearcut.

\section{Other topics}

\section{Spin Effects}

Tom Roser from BNL summarized the plans for polarized beams in RHIC. $80 \%$ polarization should be achievable. A first round of tests in the AGS looks very promising. At the high interaction energies possible in this collider, polarization effects should be relatively easy to predict in perturbative QCD. The necessary snake magnets are now part of the construction plans, so that this capability should be present at turn-on.

David Prout of Ohio State gave results for spin observables measured in an $800 \mathrm{MeV}$ proton beam at Los Alamos in ${ }^{12} \mathrm{C}(\mathrm{p}, \mathrm{n})$ reactions in the $\Delta$ resonance region. These involved both a polarized proton beam and the capability to measure the momentum and polarization of the neutron in the final state. Measurements of this type can be compared to (e,e'p) reactions, and elucidate the nuclear response functions for both transversely and longitudinally polarized protons.

\section{Exclusive Processes}

Robin Appel from Yale presented results for BNL E838. This experiment studied a wide range of exclusive processes in order to isolate the dominant quark and gluon diagrams. One conclusion is that gluon exchange does not dominate in exclusive processes, but rather quark exchange seems to be the most important mechanism.

Rex Tayloe from Ilinois presented preliminary results from LEAR experiment PS185 on $p p \rightarrow \Lambda \bar{\Lambda}, \Sigma \bar{\Sigma}$ and $\Lambda \bar{\Sigma}$. Theoretical interpretation of this data was presented by J. Speth from Institut fur Kernphysik, Julich, based on a coupled-channel meson exchange approach, which seems capable of describing the data.

\section{REFERENCES}

1. T. H. Fields and M. D. Corcoran, Phys. Rev. Lett., 70, 143 (1993).

2. M. D. Corcoran et al., Phys. Lett., B259, 209 (1991)

3. D. Naples et al., Phys. Rev. Lett., 72, 2341 (1994).

4. G. Y. Fang, FERMILAB-CONF-93-305. 